\title{
Surgical management of cervical spine deformity in chondrodysplasia punctata
}

\author{
Nobuhito Morota, MD, ${ }^{1}$ Satoshi Ihara, MD, ${ }^{1}$ Hideki Ogiwara, MD, PhD, ${ }^{2}$ and Goichiro Tamura, MD ${ }^{1}$ \\ ${ }^{1}$ Division of Neurosurgery, Tokyo Metropolitan Children's Medical Center; and 2Divison of Neurosurgery, National Center for Child \\ Health and Development, Tokyo, Japan
}

OBJECTIVE Chondrodysplasia punctata (CDP), a rare skeletal dysplasia, can lead to cervical spine instability and deformity. However, an optimal neurosurgical intervention has yet to be established. Thus, a retrospective study was conducted to assess the efficacy of various surgical interventions for children with CDP.

METHODS The authors retrospectively reviewed 9 cases of CDP in which cervical decompression with or without posterior fusion was performed between April 2007 and May 2016. Patient demographics, preoperative clinical conditions, radiographic findings, surgical procedures, and the postoperative course were analyzed in detail.

RESULTS A total of 12 operations were carried out in 9 patients ( 8 male, 1 female) during the study period. The patients' ages at the initial surgery ranged from 2 months to 2 years. Seven of the children had CDPX1, 1 had CDPX2, and 1 had tibia-metacarpal type CDP (CDP-TM). The lesion occurred at the craniovertebral junction (CVJ) in 7 cases and involved a subaxial deformity in 2 cases. The initial surgery was $\mathrm{C}-1$ laminectomy with occipitocervical fusion (OCF) followed by halo external fixation in 5 cases, OCF alone in 1 case, and C-1 laminectomy alone in 3 cases. Three children required additional surgery. In one of these cases, a staged operation was required because the patient's head was too small to attach a halo ring at the time of the initial procedure (C-1 laminectomy). In another case, OCF was performed 11 months after C-1 laminectomy because of intramedullary signal change on serial MRI, although the child remained asymptomatic. In the third case, additional posterior fusion was performed 17 months after an initial laminectomy and OCF due to newly developed cervical dislocation caudal to the original fusion. This last patient required a third operation 9 months after the second because of deep wound infection. Surgery improved the motor function of all 7 children with CDPX1, but 3 children who had already suffered respiratory failure preoperatively required continued respiratory support. At the time of this report, 7 of the 9 children were alive and in stable condition. One child died due to restrictive respiratory insufficiency, and another died in an accident unrelated to CDP.

CONCLUSIONS Surgical decompression with or without fusion for CVJ and subaxial cervical lesions in infants and toddlers with CDP generally saves lives and increases the likelihood of motor function recovery. However, in this case series the patients' preoperative condition had a strong effect on postoperative respiratory function. The surgery was not straightforward, and a second operation was required in some cases. Nevertheless, the findings indicate that early surgical intervention for CDP with cervical involvement is feasible, suggesting that the role of neurosurgery should be reevaluated.

https://thejns.org/doi/abs/10.3171/2017.5.PEDS16554

KEY WORDS chondrodysplasia punctata; surgery; cervical spine; craniovertebral junction; deformity; stenosis; posterior fusion

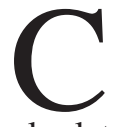
HONDRODYSPLASIA punctata (CDP) is a rare skeletal disorder stemming from a variety of causes, including genetic anomalies (peroxisomal, cholesterol related, or vitamin K metabolism related) and acquired embryopathies, such as those resulting from low maternal vitamin $\mathrm{K}$ levels or maternal use of warfarin or hydan- toin. CDP comprises a group of genetically heterogeneous skeletal dysplasias characterized by aberrant deposition of calcium (stippled epiphyses) during endochondral bone formation $^{12,26,27}$ (Fig. 1). Diagnosis is usually made in early infancy based on characteristic dwarfism, facial anomalies, and other clinical features. 


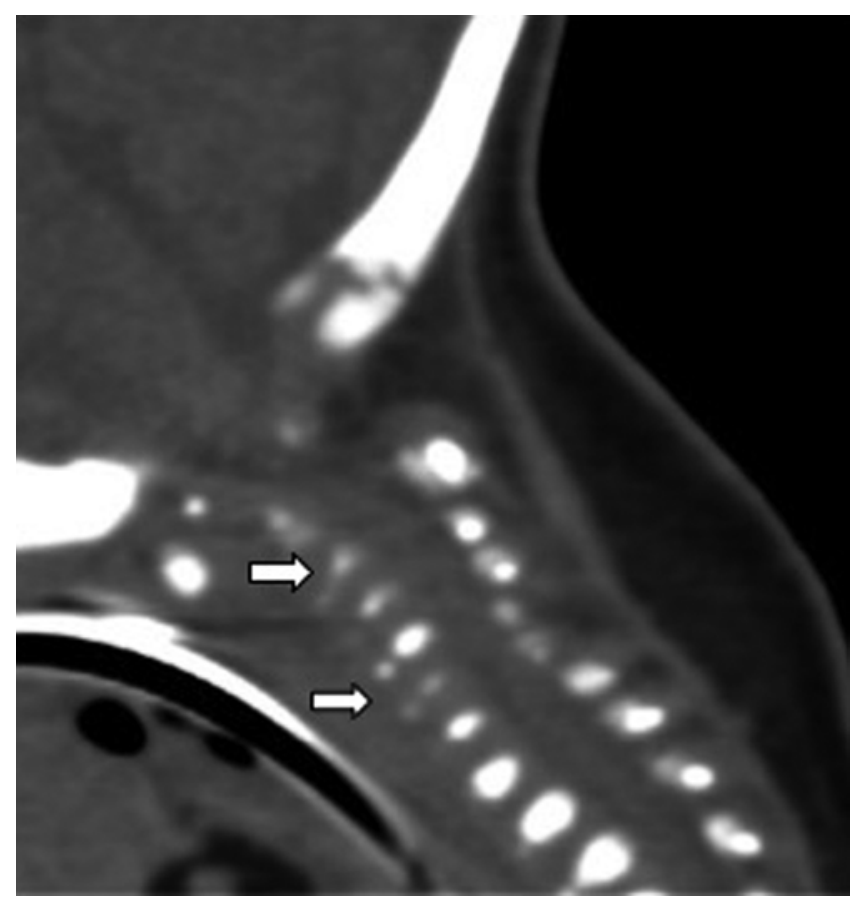

FIG. 1. Case 8. Sagittal CT reconstruction obtained in a child with CPD and cervical spine deformity showing aberrant punctiform deposition of calcium called stippled epiphyses in the cervical spine (arrows).

Because of the rare occurrence of CDP and differences in pathogenesis between the subtypes, the involvement of the central nervous system in this disease group is not fully understood. Associated cervical spine stenosis and deformity, including atlantoaxial dislocation (AAD) at the craniovertebral junction (CVJ), have been reported sporadically in a small number of patients. In their review of the English language reports, Vogel and Menezes cited a total of 44 cases with cervical spine involvement, among which only 8 cases were surgically treated (with a total of 12 operations). ${ }^{26}$ The study authors recommended conservative treatment, followed by surgery only if necessary. Surgical management has not been considered the first treatment option for any subtype of CDP due to the high risk of mortality. ${ }^{14,28,29}$
The authors had the opportunity to operate on 9 consecutive CDP patients with a CVJ and/or subaxial cervical spine deformity in the past 9 years; the lead author (N.M.) was the primary surgeon in all 9 cases. While the underlying principles governing the management of cervical stenosis with or without instability are identical, special consideration was required for the small, fragile spines of very young CDP patients. The purpose of this study was to reevaluate the role of neurosurgery by describing the surgical procedures, postoperative management, and outcomes of surgical treatment of cervical spine deformity in toddlers and infants with CDP.

\section{Methods \\ Patient Population}

Institutional review board approval was obtained before starting this retrospective study of cases involving infants and toddlers with CDP who underwent cervical spine surgery between April 2007 and May 2016. Data were collected from the patients' electronic medical records. Patient demographics, CDP subtypes, preoperative clinical condition, radiographic findings, surgical procedures, and postoperative course were reviewed in detail (Table 1). The follow-up period in Case 9 was less than 1 year. However, considering the rarity of the disease and the small number of surgically managed CDP patients, Case 9 was included in this study.

The diagnosis of CDP was made by a pediatrician or neonatologist based on clinical and radiological data before referral to the division of neurosurgery. CDP subtypes were diagnosed based on consultation with a clinical geneticist. Cervical spine involvement was suspected when a CDP patient showed motor or respiratory impairment. MRI of the brain and cervical spine, including the CVJ, was performed in all patients to locate the lesion. Cervical instability was detected using lateral flexion-extension radiographs. Once instability was suspected, CT scans were performed. Dynamic flexion-extension CT studies of the neck were carried out to assess the instability in detail and to judge its reducibility. CT sagittal and 3D reconstructions were also used to detect incomplete vertebral body formation (dysplastic vertebral body) and to select the appropriate surgical procedure ${ }^{17}$ (Fig. 2). In addition, CT angiography

TABLE 1. Initial surgical procedures in 9 CDP patients with cervical spine deformity

\begin{tabular}{ccccccl}
\hline Case No. & Age at 1st Op & Sex & CDP Subtype & Lesion & Instability & \multicolumn{1}{c}{ Details of 1st Op } \\
\hline 1 & 6 mos & M & CDP-TM & C4-5 & - & C3-5 laminectomy \\
\hline 2 & 19 mos & M & CDPX1 & C3-6 & + & O-C7 pst fusion, rib autografts \\
\hline 3 & 2 yrs & M & CDPX1 & C1-3 & + & C-1 laminectomy, O-C3 pst fusion, skull autograft \\
\hline 4 & 5 mos & M & CDPX1 & C1-2 & + & C-1 laminectomy, O-C4 pst fusion, skull autograft \\
\hline 5 & 2 mos & M & CDPX1 & C1-2 & + & C-1 laminectomy \\
\hline 6 & 2 mos & M & CDPX1 & C-1 & - & C-1 laminectomy \\
\hline 7 & 15 mos & M & CDPX1 & C1-2 & + & C-1 laminectomy, O-C4 pst fusion, skull autograft \\
\hline 8 & 12 mos & F & CDPX2 & C1-2 & + & C-1 laminectomy \\
\hline 9 & 13 mos & M & CDPX1 & C1-2 & + & C-1 laminectomy, O-C3 pst fusion, skull autograft \\
\hline
\end{tabular}

Pst $=$ posterior 

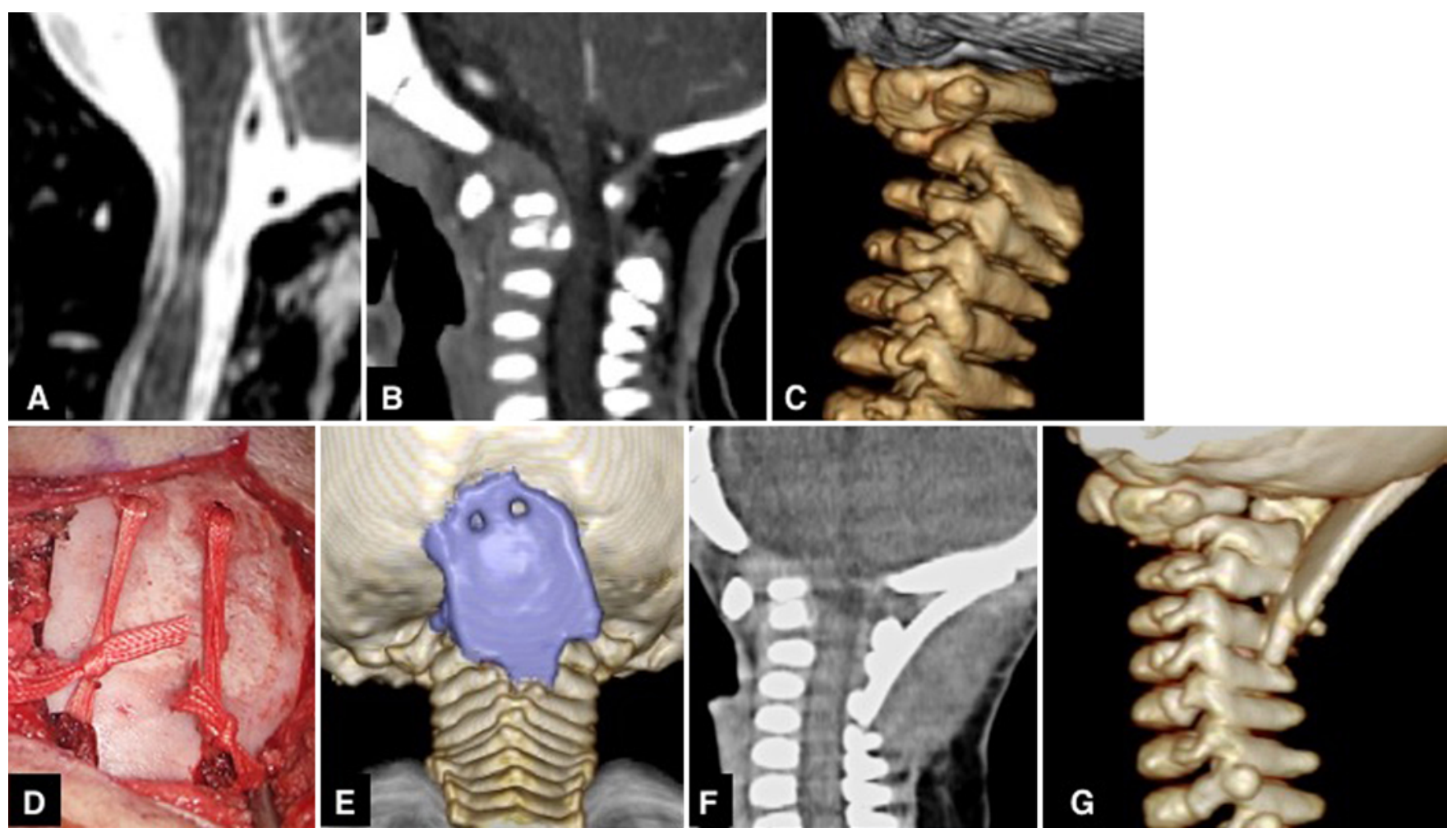

FIG. 2. Case 9. CDP associated with AAD. A: Sagittal T2-weighted MR image obtained with the neck in extension showing narrowing and intramedullary signal intensity change at the CVJ. Note that the subarachnoid space is wide in the extended neck position. B and C: Midsagittal (B) and 3D (C) CT reconstructions demonstrating CVJ stenosis in neck flexion. D: Intraoperative photograph obtained after fixing of skull autograft for O-C3 posterior fixation. A pair of UHMW-PE cables was passed from the edge of the foramen magnum to the $\mathrm{C} 2-3$ sublaminar space and then tightened. E: Postoperative 3D CT reconstruction showing the implanted skull autograft. The caudal end of the bone graft is attached to the C-4 lamina. F and G: 2D midsagittal (F) and 3D

(G) CT reconstructions demonstrating solid bony fusion 3 months after the surgery. Figure is available in color online only.

was routinely performed to detect any vascular anomalies such as a winding course or stenosis associated with CDP.

\section{Surgical Procedures and Postoperative Management}

The surgical procedures were selected based on clinical and radiological findings. Posterior cervical decompression was performed in all cases. In addition, posterior occipitocervical fusion (OCF) with use of a halo external fixation device was performed when required; OCF was considered whenever AAD was present.

The surgical protocol for OCF in CDP patients in this study includes the following steps.

1. Endonasal intubation is performed, under endoscopic guidance whenever possible.

2. A halo head ring is attached to the skull using 6 head pins applied with 1-2 lbs torque or less. ${ }^{18}$

3. Baseline motor evoked potentials (MEPs) are recorded prior to placing the patient in a prone position. After placement of the head and neck in a slightly extended position, MEPs are recorded again to confirm safety and monitored continuously thereafter.

4. During surgery, the head is placed in traction using a halo ring with a weight of one-fifth to one-tenth of the patient's body weight.

5. A bone graft for fusion is usually harvested from the skull. When a long-bone graft is required, a rib is used instead.

6. The occipitocervical region is opened. A C-1 laminectomy is performed if indicated.

7. The bone graft is fixed using an ultra-high-molecularweight polyethylene (UHMW-PE) cable system (3.0mm width, Alfresa Pharma Corp. $)^{30}$ (Fig. 2). A bur hole is opened about $1 \mathrm{~cm}$ rostral to the posterior rim of the foramen magnum. A pair of surgical threads is passed through the sublaminar space and the edge of the foramen magnum using a flexible guide wire. The UHMW-PE cable is inserted using the surgical thread as a guide. The edges of the harvested bone graft are cut partially to accommodate the cable. If the bone graft is sufficiently long, bur holes are opened on the rostral side to permit passage of the cables (Fig. 2). The bone graft is put into place, and the cable is tightened gradually using a tightening gun exclusively designed for this purpose. The head traction weights are removed before the cable is tightened. Details of the procedure for the UHMW-PE cable have been described elsewhere. ${ }^{30} \mathrm{Be}-$ cause of the unavailability of osteoinductive materials such as recombinant bone morphogenetic protein-2 in Japan, these are not used in our procedures.

8. A lateral radiograph is taken to confirm optimal alignment. 


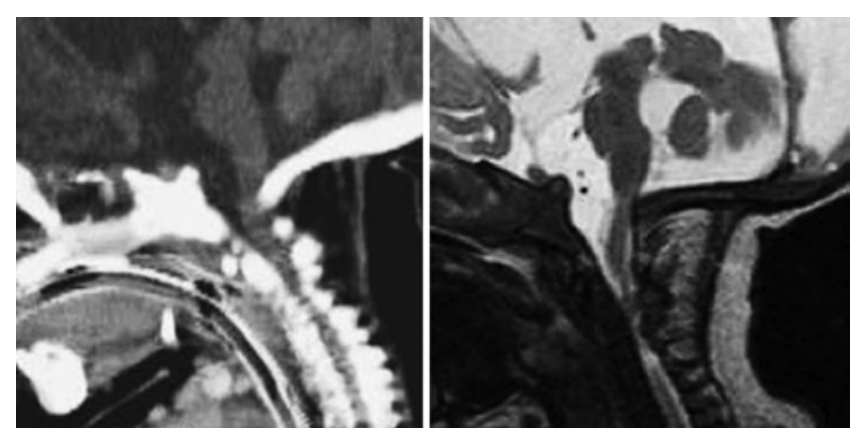

FIG. 3. Case 1. Sagittal CT reconstruction (left) and sagittal T2-weighted MR image (right) showing C4-5 stenosis in neutral neck position.

9. Following wound closure, the patient is placed in a supine position and into a halo vest. The halo ring and vest are connected, and another lateral radiograph is taken to confirm occipitocervical alignment after the OCF.

Removal of the halo orthosis was usually planned after 2.5-3 months of immobilization following OCF and performed in an operating room with the patient under general anesthesia with a laryngeal mask airway. After disconnection of the halo head ring from the vest, lateral flexion-extension radiographs were taken to confirm the stability of the CVJ before the final decision to remove the halo head ring. A cervicothoracic orthosis or cervical collar was used for external fixation for the next 3-6 months. Daily use of a cervical collar was recommended thereafter.

\section{Results}

The patients were 9 infants or toddlers ( 8 boys and 1 girl) 2 months to 2 years old (median age 13 months). The CDP subtypes were represented by 7 cases of X-linked CDP 1 (CDPX1: OMIM 302950), 1 case of X-linked CDP 2 (CDPX2: OMIM 302960), and 1 case of tibial-metacarpal (TM) type CDP (CDP-TM: OMIM 118651) (Table 1). The follow-up period after the initial surgery in the surviving children ranged from 10 to 100 months, with an average of 63 months (Table 1).

Lesion were located at the CVJ in 7 patients and the subaxial cervical spine in 2 patients. Cervical instability associated with the CVJ or subaxial cervical lesions was present in 6 patients and 1 patient, respectively. Five of these 7 children underwent OCF as an initial surgical treatment for AAD. One patient with C3-6 subaxial cervical instability had a dysplastic vertebral body and required $\mathrm{O}-\mathrm{C} 7$ posterior fusion. $\mathrm{C}-1$ laminectomy alone was per- formed as the initial procedure in 3 patients, 1 without instability and the remaining 2 with AAD. The latter 2 patients underwent $\mathrm{OCF}$ within a year after the initial surgery. One child with $\mathrm{C} 4-5$ stenosis underwent decompressive laminectomy alone (Fig. 3).

Three patients underwent a second operation (Table 2) for OCF and/or additional stabilization. In Case 5, the second operation was planned as part of a staged procedure. In Case 3, additional posterior fusion was required 17 months after the initial procedure because of progressive dislocation at $\mathrm{C} 3-4$, caudal to the originally treated levels. This patient subsequently developed a wound infection and required a third operation for removal of instrumentation (described in detail under Illustrative Cases below). In Case 8, the treatment strategy was more complex than in the other cases. Because of lack of clinical signs and symptoms and uncertainty regarding the presence of AAD, a C-1 laminectomy was performed as the initial treatment (without fusion), with the understanding that OCF would be performed as a second procedure if any clinical signs or symptoms developed. Although there were no new signs or obvious symptoms of progression, worsening of intramedullary signal hyperintensity was observed on serial imaging, leading to the decision to perform OCF 11 months after the original surgery. Fortunately, the child remained neurologically intact.

The surgical outcome was favorable in most of the children. Solid OCF was achieved in 5 of the 6 patients who underwent OCF and were followed for more than a year after the last fusion surgery. Of these, however, only 2 showed bony fusion, while the remaining 3 showed fibrous fusion. Use of a cervical collar after removal of the halo orthosis was recommended to reinforce the fragile bone tissue in the 7 children who underwent OCF.

Surgical complications were rare. A repeat fixation was carried out in 1 patient after the edge of the foramen magnum was torn during tightening of the UHMW-PE cables. Surgical site infection requiring re-exploration of the wound developed in 2 cases. Postoperative complications related to the halo orthosis were not unusual, and complications developed in 4 of the 7 children who underwent OCF. Pin site infection was the most common complication, followed by loosening of the halo ring. Two of 4 halo-related complications required reattachment of the halo ring.

No neurological deterioration attributed to surgery was observed in our series. Changes in motor function were measured using the Ability for Basic Movement Scale for Children (ABMS-C). ${ }^{19}$ The ABMS-C consists of 5 items (head control, sitting, locomotion on a flat surface, stand-

TABLE 2. Staged or additional surgery for CDP with cervical spine deformity

\begin{tabular}{ccclc}
\hline Case & Age at 2nd Op & Time Since 1st Op & \multicolumn{1}{c}{ Surgical Indication } & Surgical Procedures \\
\hline $3^{*}$ & 4 yrs & 17 mos & Progressive dislocation btwn C-3 \& C-4 & $\begin{array}{r}\text { O-C7 pst fusion, Olerud loop \& C7-T1 } \\
\text { transarticular screws, rib autografts }\end{array}$ \\
\hline 5 & 11 mos & 9 mos & Staged surgery & O-C3 pst fusion, skull autograft \\
\hline 8 & 23 mos & 11 mos & Increased intramedullary hyperintensity on MRI & O-C4 pst fusion, skull autograft \\
\hline
\end{tabular}

* This patient ultimately underwent a third operation for removal of the instrumentation due to deep wound infection 9 months after the second operation. 
ing, and walking), each scored using a scale ranging from 0 to 3 , with the result of the assessment described by scores given in order by item, followed by the total score in parentheses (Table 3). Preoperatively all but 1 patient showed impaired motor development, with 7 cases of tetraparesis and 1 case of paraparesis observed. Seven of these 8 children showed some improvement in their motor function postoperatively. One girl with CDPX2 showed normal motor development despite a signal intensity change at the C-1 level on T2-weighted MRI and remained neurologically stable postoperatively. No functional recovery was observed in 1 child with severe cervical stenosis (Table 3).

Respiratory function was severely impaired in 4 patients, 3 of whom needed mechanical respiratory support preoperatively and did not show any respiratory improvement postoperatively. One child had severe preoperative dyspnea that was managed by home oxygen therapy postoperatively. In contrast to motor function, respiratory function showed no improvement from the preoperative status in any of the patients, thus demonstrating the failure of postsurgical functional recovery. This result may be due to the fact that severely disabled children with CDP almost always experience progressive restrictive respiratory failure, and because this condition cannot be treated surgically, it normally persists even after the operation. Moreover, the cause of this condition remains unknown. In our series of patients, however, deterioration of respiratory function did not occur postoperatively and occurred in only 1 patient during follow-up (Table 3).

At the time of this report, 7 of the 9 children in this case series were still alive. One was attending special education class at regular school. The 3 other children were attending a special education school, and the remaining 3 children, who were still too young to attend school, were receiving home care from their families. One child with CDP-TM died from restrictive respiratory failure 12 months after surgery. Another child died following administration of Hib (Haemophilus influenzae type b) vaccine 18 months after surgery due to causes unrelated to the CDP.

\section{Illustrative Cases}

\section{Case 3}

This male child received the diagnosis of AAD and C-1 hypoplasia when he was 2 years old based on delayed motor development and poor head control (Fig. 4A). CDP was diagnosed after he was referred to us. Preoperatively, he was able to roll over on his bed but was unable to sit up or stand without assistance. His preoperative ABMS-C score was 0-1-1-0-0 (2). Subsequently, he underwent a C-1 laminectomy and occiput $(\mathrm{O})-\mathrm{C} 3$ posterior fusion, followed by a halo orthosis. The halo orthosis was removed 3 months later and a cervical brace was attached. Postoperatively, he showed improved upper-extremity motor function and was capable of some movement in the fingers and hands. However, serial imaging studies (radiographs and CT) during follow-up revealed progressive dislocation between using spinal instrumentation was performed when he was 4 years old (Fig. 4C), 17 months after the initial procedure. The early postoperative course was uneventful, but the paC-3 and C-4 (Fig. 4B). Occipitocervical (O-C7) fixation

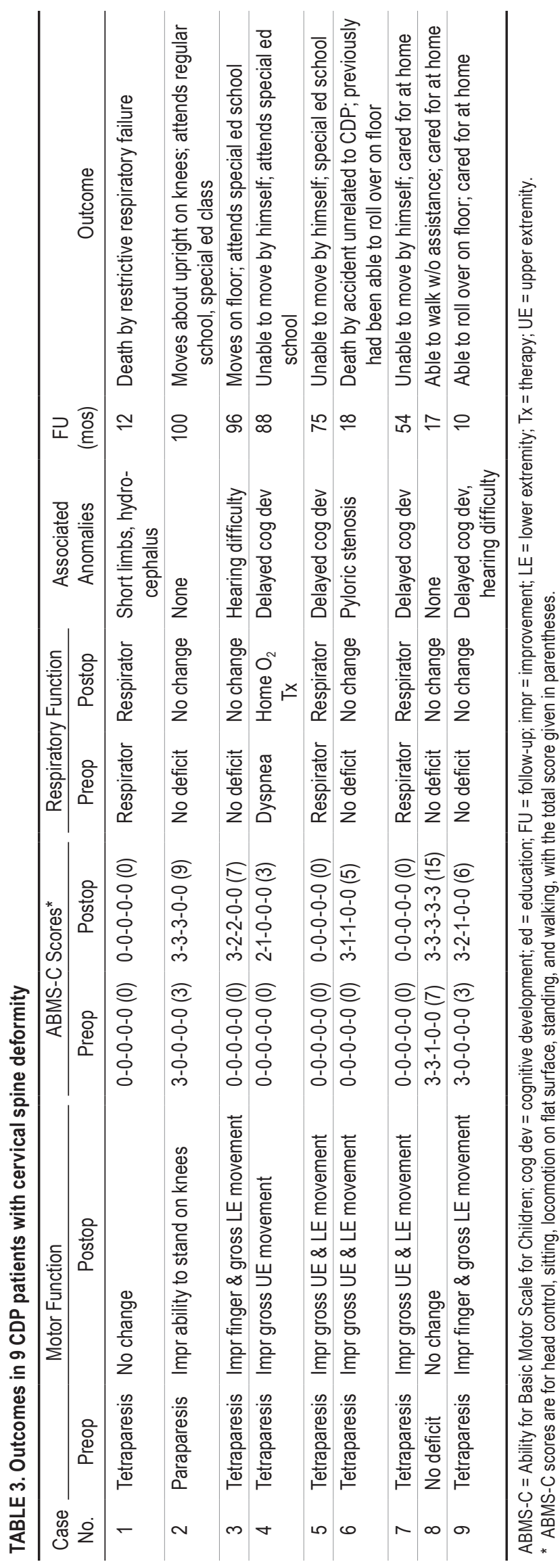



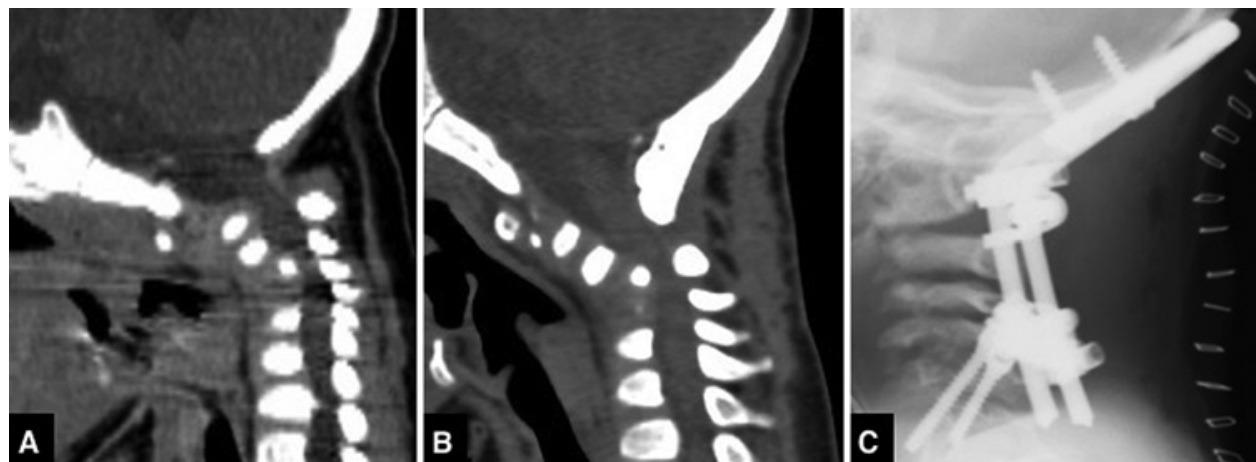

FIG. 4. Case 3. Additional surgery for progressive dislocation caudal to the OCF. A: Midsagittal CT reconstruction from preoperative study revealing severe AAD. The image shows the neck in flexion, and the CT scan was performed before the first operation when the patient was 2 years old. B: Midsagittal CT reconstruction from postoperative follow-up study showing progression of preexisting $\mathrm{C} 3-4$ dislocation progressed after $\mathrm{O}-\mathrm{C} 3$ posterior fusion. A second operation (O-C7 posterior fusion with spinal instrumentation) was scheduled for treatment of the progressive dislocation when the child was 4 years old. C: Lateral radiograph obtained after the second operation.

tient developed a deep wound infection. Because several weeks of conservative treatment failed to resolve the infection and solid bony fusion had been confirmed, the patient underwent a third operation for removal of the instrumentation 9 months after the second operation. At the time of this report, the patient was 10 years old, was attending a support school, and relied on an electric wheelchair for mobility. His latest ABMS-C score was 3-2-2-0-0 (7).

\section{Case 5}

This male child was born at 34 weeks' gestation, after receiving a prenatal diagnosis of thoracic hypoplasia, joint contraction of the extremities, and cervical spinal cord compression. ${ }^{21}$ Soon after birth he was intubated and treated with mechanical ventilation. CDP was diagnosed based on typical clinical and radiological findings. A CT scan showed marked compression at the CVJ due to $\mathrm{C}-1$ hypoplasia (Fig. 5A). AAD was also present. $\mathrm{He}$ was referred to us at 2 months of age for surgery. Because his head was too small for a halo ring attachment, staged surgery was planned, and a C-1 laminectomy alone was performed as the initial surgery. A cast specially designed to fit the dorsal cervical curvature was used for postoperative immobilization. When the patient was 11 months old and his head circumference reached $45.0 \mathrm{~cm}$, OCF was performed with placement of a halo orthosis (Fig. 5B). The halo orthosis was removed 3 months after surgery, when bony fusion was confirmed (Fig. 5C). A cervicothoracic brace was then attached. The patient showed increased spontaneous movement of the lower extremities after the first surgery, but his ABMS-C score remained $0-0-0-0-0$ (0). At the time of this report, he was attending a support school and using a portable ventilator.

\section{Case 6}

This male child was born at 37 weeks' gestation and received a postpartum diagnosis of CDP based on clinical appearance and radiological findings. Fetal ultrasonogra-
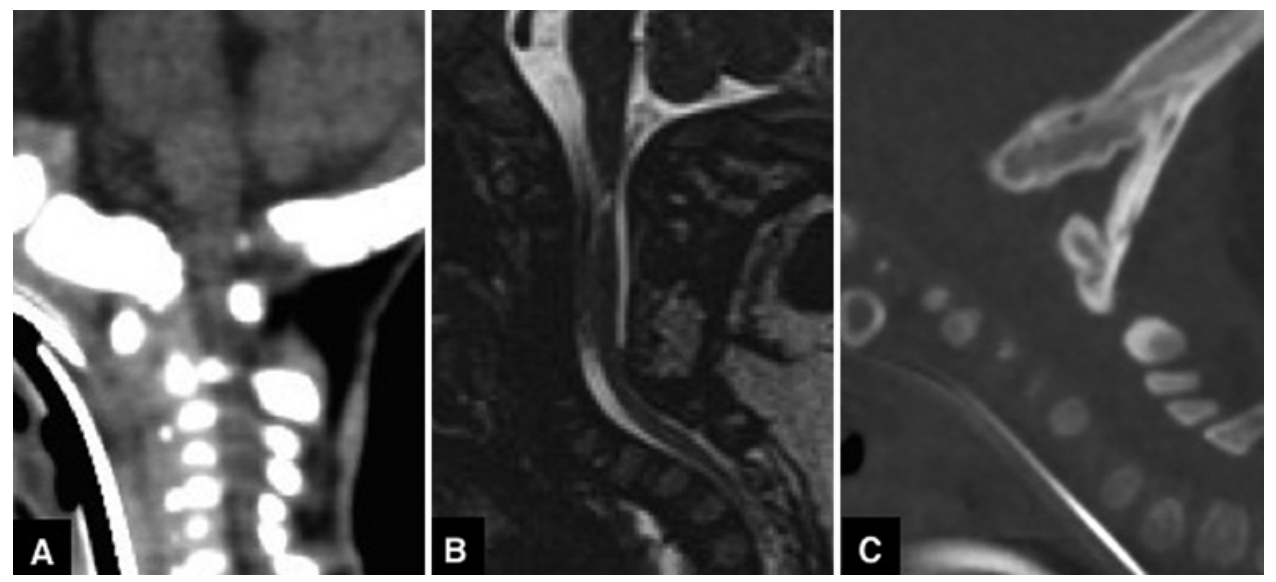

FIG. 5. Case 5. Staged surgery. A: Midsagittal CT reconstruction from study performed before the patient's first surgery at age 2 months showing C-1 hypoplasia and AAD. B: Sagittal T2-weighted MR image obtained when the patient was 11 months old revealing intramedullary signal intensity change at the CVJ. C: Midsagittal reconstruction of bone density CT scan obtained 3 months after $\mathrm{O}-\mathrm{C} 3$ posterior fusion procedure showing solid bony fusion. 


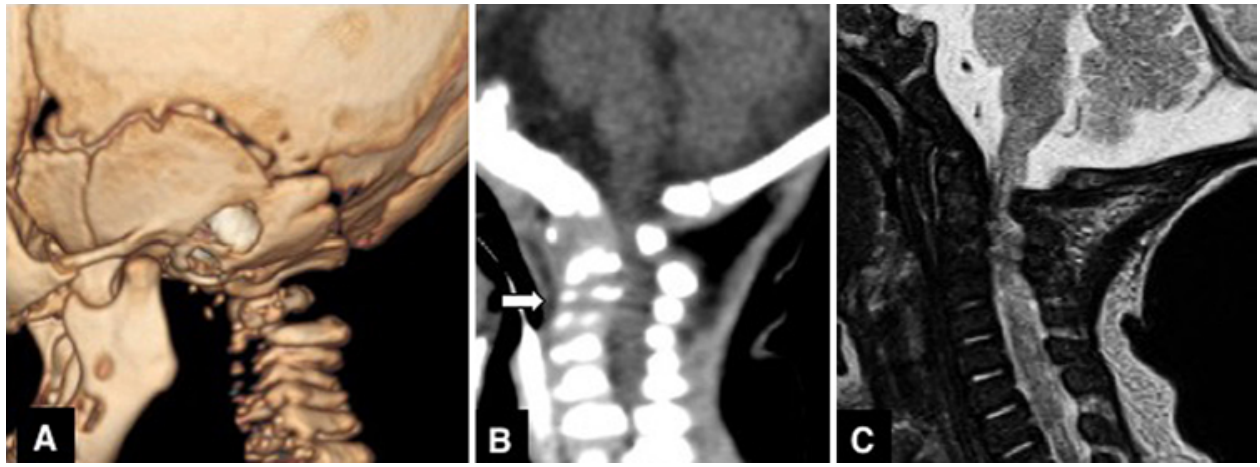

FIG. 6. Case 6. C-1 stenosis without cervical instability. A and B: Preoperative 3D (A) and sagittal (B) CT reconstructions demonstrating C-1 hypoplasia causing C-1 stenosis with spinal cord compression at the CVJ. Note the typical dysplastic findings at C-3 and C-4 (arrow). C: Sagittal T2-weighted image showing stenosis at the CVJ and cord displacement due to the hypoplastic C-1 lamina. Figure is available in color online only.

phy had revealed a flat nasal bridge at 30 weeks' gestation. Neonatal physical examination demonstrated spasticity and an abnormal ankle joint position. MRI and CT revealed marked stenosis at the CVJ due to C-1 hypoplasia (Fig. 6A-C). Despite the presence of dysplastic cervical spines, AAD was not observed. A C-1 laminectomy alone was carried out when the patient was 2 months old. His preoperative motor development was age appropriate (ABMS-C score: 0-0-0-0-0 [0]) but he demonstrated a mild delay during the postoperative follow-up examination when he was 1 year old (ABMS-C: 3-1-1-0-0 [5]). He died of causes unrelated to CDP at the age of 20 months, soon after receiving a Hib vaccination. A CT scan obtained after his death showed no lesions at the CVJ or cervical spine.

\section{Discussion}

\section{Classification of CDP}

The recent classification of CDP is based on biochemical and molecular findings. In their overview of genetic skeletal disorders, Warman et al. classified CDP into 10 subtypes based on clinical and radiographic features and molecular pathogenesis. ${ }^{27}$ Irving et al. classified CDP syndromes into 4 groups: Group 1, comprising inborn errors of metabolism; Group 2, comprising disorders of vitamin $\mathrm{K}$ metabolism; Group 3, comprising chromosomal abnormalities; and Group 4, comprising cases of unknown etiology. ${ }^{12}$ The clinical presentations and severity differed between the groups. Most children in the "inborn errors of metabolism" group had a poor survival rate while those in the "vitamin K metabolism" and "chromosomal" groups were expected to survive. ${ }^{12}$ Deformity of the vertebral body and spinal column were commonly observed in all groups but their management and clinical course were not described in detail.

In our case series, 3 subtypes of CDP were identified-namely, CDPX1, CDPX2, and CDP-TM. CDPX1 is caused by a mutation on the ARSE gene on the X chromosome (Xp22), which codes for arylsulfatase E, a Golgi enzyme; the condition is inherited as an X-linked recessive trait. ${ }^{12,21,27} \mathrm{CDPX} 1$, described as brachytelephalangic CDP in some reports, belongs to Group 2 (disorders of vi- tamin $\mathrm{K}$ metabolism) and has an estimated prevalence of 1 in 500,000 according to 1 report. ${ }^{16}$ Although the clinical course of CDPX1 is generally believed to be benign, a recent study ${ }^{8}$ documented more severe forms of this disease. Cervical spine involvement is one of the clinical features of severe CDPX1 and is present in up to $25 \%$ of CDPX1 cases. $4,8,20,26$

CDPX2, which results from defects in cholesterol synthesis stemming from a mutation on chromosome $\mathrm{X}$ (Xp11), is inherited as an X-linked dominant trait and is lethal in affected males. ${ }^{12,27}$ CDPX2 belongs to Group 1 , characterized by inborn errors of metabolism, and its clinical presentation and severity vary widely among affected female children due to different patterns of X-inactivation.? Cervical spine involvement in CDOPX2 has not been identified despite a thorough review of the literature by Vogel and Menezes. ${ }^{26}$

CDP-TM is an autosomal dominant disorder and belongs to the "unknown etiology" group. The long-term clinical course in 3 previously reported patients was favorable, with marked resolution of significant skeletal abnormalities over time. ${ }^{23}$ In the same study, 1 patient underwent a laminectomy for lumbar canal stenosis at the age of 26 years..$^{23}$ Among the other forms of CDP, spinal involvement was reported in rhizomelic CDP (R-CDP: OMIM 215100, 222765, 600121), ${ }^{14}$ an autosomal recessive disorder stemming from abnormal peroxisomal function with an incidence of 1 in 100,000 individuals. ${ }^{12,27}$ The survival rate for R-CDP was thought to be very poor for patients in their first year of life, but a recent study reported that $50 \%$ of affected children were able to survive up to 6 years of age. ${ }^{28}$ The association of cervical spine lesions does not appear to be unusual, and is expected in more than $20 \%$ of cases. ${ }^{28}$ A study of MRI features of patients with R-CDP demonstrated multiple abnormalities in association with the severe phenotype, including multilevel cervical canal stenosis without spinal cord compression. ${ }^{3}$ A case of CVJ stenosis with marked compression of the spinal cord was also reported..$^{13}$ Because of the poor prognosis associated with the severe phenotype of R-CDP, surgical intervention is rarely indicated in these cases even in the presence of severe spinal cord compression..$^{14,28,29}$ 


\section{Cervical Spine Lesions in CDP}

Spinal involvement in CDP was first described by Afshani and Girdany in 1972 in the first report of a CVJ lesion treated with decompression surgery. ${ }^{1}$ Most of the papers describing spinal lesions associated with CDP were published in the past 2 decades, after the introduction of MRI for clinical diagnosis. $3,9,10,11,13,14,17,25,29$ Vogel and Menezes, in their thorough review of the English language reports of cervical spine disease in 44 cases of CDP, stated that cervical spine disorders were most frequently encountered in R-CDP cases, followed closely by CDPX1 cases. ${ }^{26}$ In our cohort, 7 of 9 children had CDPX1, and none had R-CDP, probably because our case series was biased toward surgically treated children, while patients with cervical spine stenosis without cord compression, a common finding in $\mathrm{R}$-CDP, were not included. ${ }^{14,28}$ CDPX2 was rarely associated with cervical spine abnormalities. ${ }^{26}$ Typical spinal lesions in CDPX2 were located at the thoracolumbar spine and presented kyphoscoliosis..$^{15,17}$

\section{Indications for Surgery}

To date, no indications for surgical intervention or criteria for surgical procedures have been proposed for the treatment of cervical spine deformity in CDP, even for patients with severe spinal cord compression and accompanying neurological impairment. ${ }^{13,14,28,29}$ According to Vogel and Menezes, "only eight patients (29\%) of the 28 cases with reported treatment data had operative intervention."26

Based on our limited experience, we believe that surgical decompression and fusion for CVJ and subaxial cervical lesions in CDP are lifesaving procedures that can also improve the patient's condition. However, some caveats are in order when considering the indications for surgery. First, surgery is strongly recommended if stenotic lesions or any instability responsible for the clinical symptoms is present. Second, if a change in the pathological signal intensity on the MRI is observed at the site of stenosis, surgery should be considered even if the patient is asymptomatic. Third, indications for surgery should be carefully considered if severe restrictive respiratory insufficiency is present or the patient's general condition has seriously deteriorated.

Diagnosis of CDP subtypes does not preclude surgical intervention. Our knowledge regarding CDP is still limited, but data accumulated thus far revealing new aspects of the disease suggest that surgical indications for cervical spine deformity in CDP need to be individualized. ${ }^{28}$ Even for R-CDP, with its dismal prognosis, surgery is an option if the case fulfills the conditions described above. The most common causes of death in R-CDP are secondary respiratory complications. ${ }^{28}$ If the respiratory problem is caused by spinal cord compression, rather than by restrictive pulmonary impairment, surgery can enable stabilization of the compromised respiratory function in some patients.

Final decisions regarding surgical indications must be based on weighing the risks and benefits to the patients, including the possibility of surgical morbidity, economical considerations related to treatment, and the long-term prognosis. A more conservative approach might be preferable in some cases.

\section{Surgical Procedure}

Previous reports have presented OCF or decompression alone for CVJ lesions $s^{1,6,11,17,24,25}$ and a laminectomy alone for subaxial stenosis ${ }^{6,11}$ as the only feasible surgical options. The anterior approach was chosen in 2 cases in which the main pathology occurred in the anterior structures..$^{24,25}$ Once the type of surgery was decided upon, the process of selecting the specific surgical procedure was identical with that for other CVJ pathologies. ${ }^{2,18}$

The association with dysplastic vertebral bodies (and the clivus at the $\mathrm{CVJ}$ ) presents a unique feature of cervical spine deformity in CDP. Diagnosis of AAD is not straightforward in CDP because dysplasia of C-1 and C-2 often prevents standard measurements of the atlantodental interval. The presence of AAD can be comprehensively assessed based on the anatomical relationship between the remaining bony structures. Based on these findings, cervical spine stenosis in CDP can be classified as follows: Group 1a, CVJ lesion with instability; Group 1b, CVJ lesion without instability; Group 2a: subaxial lesion with dysplastic vertebral body; and Group $2 b$, subaxial lesion without dysplastic vertebral body.

Because the presence of a dysplastic vertebral body tends to lead to instability, spinal fusion surgery together with decompression for stenosis is indicated. Theoretically, the following surgical options exist: for Group 1a, posterior decompression (C-1 laminectomy, FMD) with OCF; for Group 1b, posterior decompression alone; for Group 2a, anterior decompression and fusion; and for Group 2b, posterior decompression.

In our series, 6 of 7 children with CVJ lesions in Group 1a underwent a C-1 laminectomy and OCF (Fig. 4). One child in Group 1b underwent a C-1 laminectomy alone. Another child in Group 2a received an OCF because the anterior lesion extended from C-3 to C-7, rendering an anterior fixation impossible. Furthermore, an anterior corpectomy with fusion was not a common practice in pediatric neurosurgery until recently. ${ }^{8}$ The last child in Group $2 \mathrm{~b}$ underwent posterior decompression alone.

\section{Surgical Considerations and Pitfalls}

A strategic approach is necessary for certain patients with CDP. In our series, 2 patients required staged operations. (Details of the clinical course were described previously.) A staged operation should be considered for OCF in pediatric patients with a small head size as well as patients whose general condition or comorbidities disallow lengthy, complicated procedures. Candidates for staged operations have to be selected carefully, however, as immobilization of the neck during the period between the surgical procedures is difficult to achieve in infants and young children.

Surgical technique must be considered carefully once the surgical strategy has been decided. The types of instruments used in the surgical treatment of cervical spine deformities in CDP have not been discussed in detail. Bone fragility caused by biosynthetic abnormality makes stable fixation with a rigid internal fixation construct difficult, although this method is gaining acceptance as a new procedure for use with pediatric patients with CVJ lesions. ${ }^{2}$ Nevertheless, lack of evidence for the safety of 
the procedure as well as our own lack of experience with spinal instrumentation in very young children were deciding factors in our choice of traditional OCF with halo orthoses in the present study. In our case series, spinal instrumentation was only used in 1 case (Case 3), and it was only used when the patient underwent reoperation for progressive dislocation at the age of 4 years (Fig. 4). Although instrumentation in surgery seems more common for thoracolumbar fixation for kyphoscoliosis in CDP, the average age of the children around 5 years, older than our patients or those in other studies with cervical spine involvement. ${ }^{15,17,26}$ So far, there has been only one case of instrumentation performed on a 10-month-old child with CDP reported in a case series of pediatric CVJ fixation using C-2 laminar screws. ${ }^{22}$ Until we accumulate more experience and evidence, a conservative procedure for this particular pathology in very young children is preferable.

To secure the implanted bone grafts, we routinely used a 3-mm-wide tape-like UHMW-PE cable, which allows us to secure the bone graft to the graft bed more gently than would be possible with metallic cables.$^{30}$ In the present case series, UHMW-PE was used for 7 children in 8 OCF procedures (Fig. 2). No loosening or deterioration of the cable, or fracturing of the implanted bone graft during the postoperative course, was observed.

The halo orthosis should be used with care in treating patients with CDP, as the abnormal fragility of the bone requires the application of minimal pin pressure. ${ }^{5}$ Menezes recommended the use of 8 pins with a pin pressure "controlled with thumb and forefinger pressure that generates between 1 and $11 / 3$ lbs torque" in children below the age of 2-4 years. ${ }^{18}$ Our protocol involves using 6 head pins applied with approximately $1-2$ lbs torque or less in children with CDP. Thus far, there have been no instances of skull fractures or pin penetration deep inside the skull. However, loosening or slippage of the halo ring was observed, preceded by pin site infection or similar complications. When a skin infection is present, changing the pin site is recommended.

Long-term follow-up studies of surgically treated children with CDP and cervical spine deformity are few. ${ }^{1,6,11,17,24-26}$ Data obtained from observations of thoracolumbar fusion suggest a high incidence of progressive deformity even after posterior fusion, often requiring multiple additional surgeries. ${ }^{14,15,26}$ This progressive, postsurgical deformity is attributable to the fragility of the abnormal bone in CDP, in which fibrous fusion by pseudarthrosis develops faster than the adjacent segment, such that even the grafted bone can eventually deform. In addition, the presence of a dysplastic anterior structure can accelerate deformity. An anterior approach for grafting is therefore necessary for CDP patients in Groups 1a and $2 \mathrm{a}$ when the children are mature enough to undergo the procedure. $^{15,17}$ The follow-up period in the present study was short, with only 1 child requiring additional surgery for adjacent-segment deformity after initial fusion surgery. Vigilant long-term follow-up is crucial. In addition, the daily use of a cervical collar, even after removal of a halo device, may help to prevent postoperative deformities in patients with CDP.

\section{Limitations}

This was a retrospective study with a limited number of patients. The cohort was biased for CDPX1, and the primary lesion was located at the CVJ. The follow-up period was insufficient. The results and speculations presented may not apply to other subtypes of CDP.

\section{Conclusions}

Surgical decompression with or without fusion for CVJ and subaxial cervical lesions in CDP can be a lifesaving procedure capable of restoring motor function. The diagnosis of CDP did not preclude surgery. The motor function had a higher probability of recovery with surgery than respiratory function did, with improvements in respiratory function depending on the patients' preoperative condition. Surgery was not straightforward. The fragility of the abnormal bone in CDP raised unique long-term problems requiring staged surgery or additional unplanned operations in some cases. The use of spinal instrumentation and anterior fusion surgery for this disease in the location described needs to be further assessed. From our limited experience, however, early intervention for CDP with cervical involvement seems feasible, and the role of neurosurgery needs to be reevaluated.

\section{Acknowledgments}

We thank Mr. James Robert Valera for his editorial assistance and professional advice on the manuscript. We also appreciate Dr. Ken-ichi Usami, Division of Neurosurgery, National Center for Child Health and Development, for his assistance in preparing and correcting the clinical data.

\section{References}

1. Afshani E, Girdany BR: Atlanto-axial dislocation in chondrodysplasia punctata. Report of the findings in two brothers. Radiology 102:399-401, 1972

2. Anderson RCE, Ragel BT, Mocco J, Bohman LE, Brockmeyer DL: Selection of a rigid internal fixation construct for stabilization at the craniovertebral junction in pediatric patients. J Neurosurg 107:36-42, 2007

3. Bams-Mengerink AM, Majoie CBLM, Duran M, Wanders RJA, Van Hove J, Scheurer CD, et al: MRI of the brain and cervical spinal cord in rhizomelic chondrodysplasia punctata. Neurology 66:798-803, 789, 2006

4. Braverman NE, Bober M, Brunetti-Pierri N, Oswald GL: Chondrodysplasia punctata 1, X-linked, in Pagon RA, Adam MP, Ardinger HH, et al (eds): GeneReviews. Seattle: University of Washington, 2014

5. Dormans JP, Criscitiello AA, Drummond DS, Davidson RS: Complications in children managed with immobilization in a halo vest. J Bone Joint Surg Am 77:1370-1373, 1995

6. Eash DD, Weaver DD, Brunetti-Pierri N: Cervical spine stenosis and possible vitamin $\mathrm{K}$ deficiency embryopathy in an unusual case of chondrodysplasia punctata and an updated classification system. Am J Med Genet A 122A:70-75, 2003

7. Feldmeyer L, Mevoarh B, Grzeschik KH, Huber M, Hohl $\mathrm{D}$ : Clinical variation in X-linked dominant chondrodysplasia punctata (X-linked dominant ichtyosis). Br J Dermatol 154:766-769, 2006

8. Garber ST, Brockmeyer DL: Management of subaxial cervical instability in very young or small-for-age children using a static single-screw anterior cervical plate: indications, results, and long-term follow-up. J Neurosurg Spine 24:892-896, 2016 
9. Garnier A, Dauger S, Eurin D, Parisi I, Parenti G, Garel C, et al: Brachytelephalangic chondrodysplasia punctata with severe spinal cord compression: report of four new cases. Eur J Pediatr 166:327-331, 2007

10. Goodman P, Dominguez R: Cervicothoracic myelopathy in Conradi-Hunermann disease: MRI diagnosis. Magn Reson Imaging 8:647-650, 1990

11. Herman TE, Lee BCP, McAlister WH: Brachytelephalangic chondrodysplasia punctata with marked cervical stenosis and cord compression: report of two cases. Pediatr Radiol 32:452-456, 2002

12. Irving MD, Chitty LS, Mansour S, Hall CM: Chondrodysplasia punctata: a clinical diagnostic and radiological review. Clin Dysmorphol 17:229-241, 2008

13. Jurkiewicz E, Marcinska B, Bothur-Nowacka J, Dobrzanska A: Clinical and radiological pictures of two newborn babies with manifestations of chondrodysplasia punctata and review of available literature. Pol J Radiol 78:57-64, 2013

14. Khanna AJ, Braverman NE, Valle D, Sponseller PD: Cervical stenosis secondary to rhizomelic chondrodysplasia punctata. Am J Med Genet 99:63-66, 2001

15. Lykissas MG, Sturm PF, McClung A, Sucato DJ, Riordan M, Hammerberg KW: Challenges of spine surgery in patients with chondrodysplasia punctata. J Pediatr Orthop 33:685693, 2013

16. Malou E, Gekas J, Troucelier-Lucas V, Mornet E, Razafimanantsoa L, Cuvelier B, et al: [X-linked recessive chondrodysplasia punctata. Cytogenetic study and role of molecular biology.] Arch Pediatr 8:176-180, 2001 (Fr)

17. Mason DE, Sanders JO, MacKenzie WG, Nakata Y, Winter $\mathrm{R}$ : Spinal deformity in chondrodysplasia punctata. Spine (Phila Pa 1976) 27:1995-2002, 2002

18. Menezes AH: Decision making. Childs Nerv Syst 24:11471153,2008

19. Miyamura K, Hashimoto K, Honda M: Validity and reliability of Ability for Basic Movement Scale for Children (ABMSC) in disabled pediatric patients. Brain Dev 33:508-511, 2011

20. Nino M, Matos-Miranda C, Maeda M, Chen L, Allanson J, Armour C, et al: Clinical and molecular analysis of arylsulfatase $\mathrm{E}$ in patients with brachytelephalangic chondrodysplasia punctata. Am J Med Genet A 146A:997-1008, 2008

21. Ochiai D, Takamura K, Nishimura G, Ikeda T, Yakubo K, Fukuiya T: Prenatal diagnosis of cervical spinal cord compression in chondrodysplasia punctata brachytelephalangic type: a case report and literature review. Congenit Anom (Kyoto) 53:160-162, 2013

22. Savage JG, Fulkerson DH, Sen AN, Thomas JG, Jea A: Fixation with C-2 laminar screws in occipitocervical or C1-2 constructs in children 5 years of age or younger: a series of 18 patients. J Neurosurg Pediatr 14:87-93, 2014
23. Savarirayan R, Boyle RJ, Masel J, Rogers JG, Sheffield LJ: Longterm follow-up in chondrodysplasia punctata, tibiametacarpal type, demonstrating natural history. Am J Med Genet A 124A:148-157, 2004

24. Takano H, Smith WL, Sato Y, Kao SC: Cervical spine abnormalities and instability with myelopathy in warfarinrelated chondrodysplasia: 17-year follow-up. Pediatr Radiol 28:497-499, 1998

25. Violas P, Fraisse B, Chapuis M, Bracq H: Cervical spine stenosis in chondrodysplasia punctata. J Pediatr Orthop B 16:443-445, 2007

26. Vogel TW, Menezes AH: Natural history and management of cervical spine disease in chondrodysplasia punctata and coumarin embryopathy. Childs Nerv Syst 28:609-619, 2012

27. Warman ML, Cormier-Daire V, Hall C, Krakow D, Lachman R, LeMerrer M, et al: Nosology and classification of genetic skeletal disorders: 2010 revision. Am J Med Genet A 155A:943-968, 2011

28. White AL, Modaff P, Holland-Morris F, Pauli RM: Natural history of rhizomelic chondrodysplasia punctata. Am J Med Genet A 118A:332-342, 2003

29. Yang BP, Mindea SA, DiPatri AJ Jr: Cervical spinal cord compression in chondrodysplasia punctata. Case illustration. J Neurosurg 104 (3 Suppl):212, 2006

30. Yonezawa I, Arai Y, Tsuji T, Takahashi M, Kurosawa H: Atlantoaxial transarticular screw fixation and posterior fusion using ultra-high-molecular-weight polyethylene cable. J Spinal Disord Tech 18:392-395, 2005

\section{Disclosures}

The authors report no conflict of interest concerning the materials or methods used in this study or the findings specified in this paper.

\section{Author Contributions}

Conception and design: Morota. Acquisition of data: all authors. Analysis and interpretation of data: Morota, Tamura. Drafting the article: Morota. Critically revising the article: Ogiwara, Tamura. Reviewed submitted version of manuscript: all authors. Approved the final version of the manuscript on behalf of all authors: Morota. Administrative/technical/material support: Morota. Study supervision: Morota.

\section{Correspondence}

Nobuhito Morota, Tokyo Metropolitan Children's Medical Center, Neurosurgery, 2-8-29 Musashi-dai, Fuchu, Tokyo 183-8561, Japan. email: nobu.m01@gmail.com. 